\title{
Notch mediates the sprouting of tip cells
}

DOI:

10.1038/nrg2081

URLs
During sprouting angiogenesis, tip cells are selected from the endothelial cells of existing vessels to pave the way for new blood vessels. Although a gradient of vascular endothelial growth factor A (VEGFA) has been shown to influence the selection and to guide the routes of these tip cells, the mechanism that regulates how many and which cells are selected to become tip cells has remained elusive. Three studies have examined these issues and have established that Notch signalling mediates the selection of tip cells during angiogenesis.

Hellström and colleagues focused on the role of Notch during angiogenesis in mouse retina. The administration of $\gamma$-secretase inhibitors (GSIs) that potently inhibit Notch to mice during the first postnatal week resulted in increased vascular density. GSI-induced Notch inhibition also resulted in more cells containing the tip-cell marker platelet-derived growth factor $B$ (PDGFB). Together these morphological and gene expression data indicate that the inhibition of Notch causes an increase in the number of tip cells, resulting in increased vascular sprouting. Conversely, treatment with a Notch agonist showed that increased Notch activity results in decreased vascular density and sprouting.

Localization studies using mice in which cells with active Notch are labelled with GFP showed that delta-like 4 (Dll4), a Notch substrate, colocalized with Notch activity at the sprouting front. Furthermore, $96 \%$ of tip cells showed intracellular accumulation of Dll4, indicative of exposure to the Notch signal. By contrast, Dll4 did not accumulate in cells behind the sprouting front. The importance of Notch-Dll4 signalling was further supported with the finding that $\mathrm{Dll} 4^{+/-}$mice had similar phenotypes to mice treated with the Notch-inhibiting GSIs.

Similar findings were reported by Siekmann and Lawson, who used transgenic zebrafish with fluorescently labelled blood vessels to study tip-cell

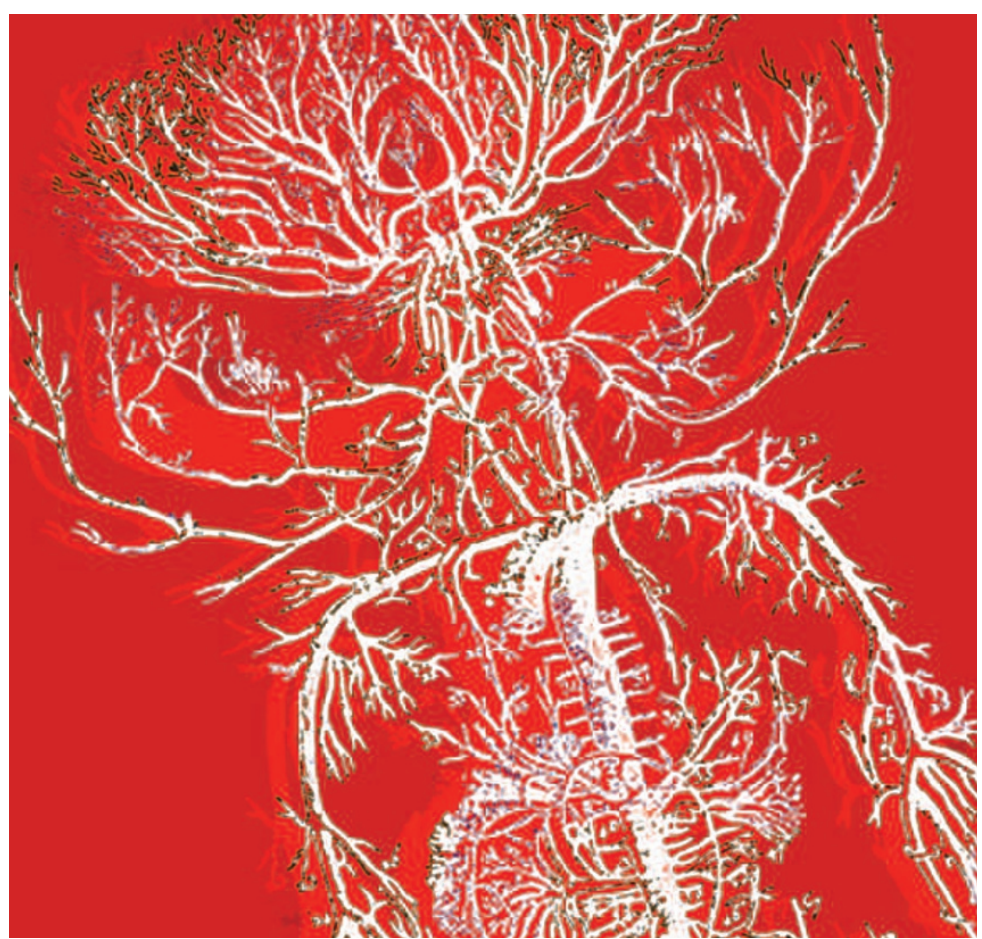

selection. Morpholinomediated knockdown of Rbpsuh (a DNA-binding protein that mediates the transcription of Notch target genes) increased blood-vessel sprouting and the number of cells that showed tip-cell behaviour. By contrast, the activation of Notch blocked blood-vessel sprouting. Also, the morpholino-induced loss of Dll4 phenocopied the loss of Notch activity, although the decreased severity of the morphological defects that were associated with the lack of Dll4 indicated that other Notch substrates might be involved in blood-vessel sprouting.

Having also found a similar role for Notch-Dll4 signalling in zebrafish, Leslie and co-workers further examined the relationship between this signalling pathway and Vegfa. They showed that the treatment of embryos that lack Dll4 (and therefore Notch signalling) with an inhibitor of Vegfa prevented aberrant blood-vessel sprouting.

Together, these data indicate that whereas VEGFA promotes the sprouting of tip cells, Notch-Dll4 signalling dampens responsiveness to VEGFA in some cells but not in others, thereby mediating tip-cell selection. As pointed out by Hellström and colleagues, this could mean that inhibitors and modulators of this Notch-Dll4 pathway could prove to be useful for the treatment of pathological angiogenesis in tumours, for which VEGFA inhibition is currently the only approved strategy.

Asher Mullard, Senior Copy Editor, Nature Reviews Molecular Cell Biology

ORIGINAL RESEARCH PAPERS

Hellström, M. et al. Dll4 signalling through Notch1 regulates formation of tip cells during angiogenesis. Nature 28 January 2007 (doi:10.1038/nature05571) |Siekmann, A. F. \& Lawson, N. D. Notch limits angiogenic cell behaviour in developing zebrafish arteries. Nature 28 January 2007 (doi:10.1038/ nature05577) | Leslie, J. D. et al. Endothelial signalling by the Notch ligand Delta-like 4 restricts angiogenesis. Development 134 839-844 (2007) 